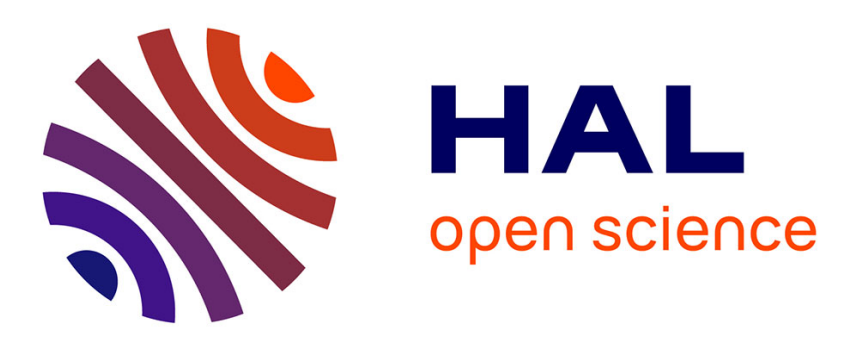

\title{
Some ring-shaped potentials as a generalized 4-D isotropic oscillator. Periodic orbits
}

\author{
Eva Tresaco, Sebastián Ferrer
}

\section{To cite this version:}

Eva Tresaco, Sebastián Ferrer. Some ring-shaped potentials as a generalized 4-D isotropic oscillator. Periodic orbits. Celestial Mechanics and Dynamical Astronomy, 2010, 107 (3), pp.337-352. 10.1007/s10569-010-9258-6 . hal-00552501

\section{HAL Id: hal-00552501 https://hal.science/hal-00552501}

Submitted on 6 Jan 2011

HAL is a multi-disciplinary open access archive for the deposit and dissemination of scientific research documents, whether they are published or not. The documents may come from teaching and research institutions in France or abroad, or from public or private research centers.
L'archive ouverte pluridisciplinaire HAL, est destinée au dépôt et à la diffusion de documents scientifiques de niveau recherche, publiés ou non, émanant des établissements d'enseignement et de recherche français ou étrangers, des laboratoires publics ou privés. 


\title{
Some ring-shaped potentials as a generalized 4-D isotropic oscillator. Periodic orbits
}

\author{
Eva Tresaco - Sebastián Ferrer
}

Received: 9 December 2008 / Revised: 19 December 2009 / Accepted: 2 February 2010 /

Published online: 18 May 2010

(C) Springer Science+Business Media B.V. 2010

\begin{abstract}
A generalized integrable biparametric family of 4-D isotropic oscillators is proposed. It allows to treat in a unified way, Pöschl-Teller, Hartmann and other ring-shaped systems. This approach, based in the use of two canonical extensions, helps to simplify the studies of classical aspects of those systems. As an illustration, an analysis of the periodic solutions of those system is presented.
\end{abstract}

Keywords 4-D isotropic oscillators · Generalized Hartmann potential · Ring-shaped systems $\cdot$ Pöschl-Teller potential $\cdot$ Superintegrability

\section{Introduction}

This paper deals with with a 4-D integrable dynamical system defined by the parametric Hamiltonian function

$$
\mathcal{H}_{\mathcal{O}}=\frac{1}{2}\left(Q_{1}^{2}+Q_{2}^{2}+Q_{3}^{2}+Q_{4}^{2}+\omega\left(q_{1}^{2}+q_{2}^{2}+q_{3}^{2}+q_{4}^{2}\right)+\frac{a}{q_{1}^{2}+q_{2}^{2}}+\frac{b}{q_{3}^{2}+q_{4}^{2}}\right)
$$

(where $\omega, a$ and $b$ are parameters), and its relation with two families of 3-D integrable Hamiltonian systems $\mathcal{H}=\frac{1}{2}\|X\|^{2}+V_{i}$ with axial symmetry, namely systems with potentials given by

$$
V_{1}=-\frac{\mu}{\sqrt{x_{1}^{2}+x_{2}^{2}+x_{3}^{2}}}+\frac{P}{x_{1}^{2}+x_{2}^{2}}+\frac{Q x_{3}}{\left(x_{1}^{2}+x_{2}^{2}\right) \sqrt{x_{1}^{2}+x_{2}^{2}+x_{3}^{2}}},
$$

E. Tresaco $(\varangle)$

Dpto. de Matemática Aplicada, Universidad de Zaragoza, 50009 Zaragoza, Spain e-mail: etresaco@unizar.es

S. Ferrer

Dpto. de Matemática Aplicada, Universidad de Murcia, 30071 Espinardo, Spain

e-mail: sferre@um.es 
dubbed as Smorodinsky-Winternitz potential (see Mardoyan 2003), and

$$
V_{2}=\frac{\Omega^{2}}{2}\left(x_{1}^{2}+x_{2}^{2}+x_{3}^{2}\right)+\frac{P}{2 x_{3}^{2}}+\frac{Q}{2\left(x_{1}^{2}+x_{2}^{2}\right)},
$$

(where $\mu, \Omega, P$ and $Q$ are parameters). Note that, written in spherical variables, potentials $V_{1}$ also appear in the literature under the Pöschl-Teller form

$$
V_{1}=-\frac{\mu}{r}+\frac{P+Q}{4 r^{2} \sin ^{2} \frac{\phi}{2}}+\frac{P-Q}{4 r^{2} \cos ^{2} \frac{\phi}{2}} .
$$

The particular case of the system (1) when $a=b$ was considered by Kibler and Negali (1984a) when they studied the Hartmann potential using the Kustaanheimo-Stiefel transformation. In this sense, the proposed Hamiltonian (1) represents a generalization of theirs.

Potentials $V_{i}$ belong to a larger family of integrable systems which are known to be separable (Makarov et al. 1967). These potentials have received special attention since the pioneer work of Hartmann et al., due to its relation to the benzene molecule, as well as other models in quantum chemistry and nuclear physics. When we take $Q=0$ in potential $V_{1}$ we have the Hartmann (1972) model. Continuing the study done by Kibler and Negali (1984a), the solution was detailed by Kibler and Winternitz (1987) in parabolic coordinates. With respect to potential $V_{2}$, the case $P=0$ has been studied by Quesne (1988). Finally, Kibler et al. (1992) developed a complete study of both potential models.

The ring-shaped features come from the fact that coefficients have to be taken within specific ranges. Systems defined by these potentials are super-integrable but not maximally super-integrable, having four globally defined single-valued integrals of motion. They admit two maximally super-integrable systems as limiting cases, viz, the Coulomb-Kepler system and the isotropic harmonic oscillator system in three dimensions. This is related with the fact that Schrödinger equation is separable, among others, in spherical, parabolic and spheroidal coordinates. All finite trajectories are quasi-periodic; they become truly periodical if a commensurability condition is imposed on an angular momentum component. Kibler et al. (1994) studied in detail the coefficients of the interbasis expansions between three bases (spherical, parabolic and spheroidal) in the case of potential $V_{1}$. For the path integral approach applied to these and related systems we mention the review paper of Grosche (1992) and references therein. Recently the normalized wavefunctions and explicit expressions for their radial average values have been presented by Chen et al. (2002), where an updated list of references on these problems is given. Similar studies for potential $V_{2}$ were done by Kibler and Winternitz (1990), and Kibler et al. in 1996.

This paper deals with the relation of these 3-D systems with a 4-D integrable dynamical system defined by the Hamiltonian function (1), focused on aspects related to classical dynamics. With respect to quantum mechanics approach, we refer to the classic paper of Calogero (1969) (Sect. 2) which, with minor changes, can be applied to the two coupled one degree of freedom systems defining our model.

Also, it is presented a description of the periodic solutions found. The role of periodic orbits in Hamiltonian systems was first recognized by Poincaré (1892), stating that periodic orbits are the only opening through which we may try to penetrate the stronghold when studying complex dynamical systems. Deprit and Henrard (1967) also considered periodic solutions as the skeleton around which the dynamics is organized. Accordingly, we may find many studies in the literature focused on the analysis of periodic trajectories in order to get information of the behavior of the dynamical model. Today periodic orbits are at the basis 
of both classical and quantum mechanics. See, for example, recent works by Bhaduri et al. (2005), Palacián (2007) and Hadjidemetriou (2008).

It is clear that, like any other superintegrable system, the system defined by the Hamiltonian function (1) opens different lines for analysis: analytical, numerical, geometric and topological. For a general view on this the reader may consult Fassò (2005) and references therein. Here, having established the connection among the 3-D and 4-D models Ferrer (2006), we focus on conditions for periodic orbits. Geometric properties connected to invariant manifolds as well as symmetries of the system, which complement the work presented here, make the content of another paper (see Balsas et al. 2009).

This paper is organized as follows. In Sect. 2, assuming $\omega>0$, we carry out the integration of the system defined by Hamiltonian (1), which is given by means of elementary functions. Section 3 presents an analysis of some families of periodic orbits. In Sect. 4 we establish the relation between the oscillator and the systems defined by potentials (2) and (3) respectively, making use of well known point transformations in 4-D and their canonical extensions; for each case there is a linear system which relates parameters $P$ and $Q$ of the potentials with integrals and parameters of the 4-D oscillator.

\section{The biparametric oscillator and its integration}

Prior to show the relation of both families of ring-shaped systems with the system defined by the Hamiltonian function (1), we focus first on the integration of our oscillator. The Hamiltonian function (1) defines an integrable system in $\Delta=\mathbb{R}^{4}-\left\{(0,0) \times \mathbb{R}^{2}\right\} \bigcup\left\{\mathbb{R}^{2} \times(0,0)\right\}$.

\subsection{Still another canonical extension. Superintegrability}

There is a large literature on the issue of integrability and superintegrability which we do not consider necessary to treat here. We wish only mention that Liouville-Arnold conditions for integrability are satisfied for our system.

Let us consider a canonical extension, the polar-polar transformation $\left(q_{1}, q_{2}, q_{3}, q_{4}\right) \rightarrow$ $\left(\rho_{1}, \rho_{2}, \alpha_{1}, \alpha_{2}\right)$, considered previously by Kibler and Winternitz (1987), and given by

$$
q_{1}=\rho_{1} \cos \alpha_{1}, \quad q_{2}=\rho_{1} \sin \alpha_{1}, \quad q_{3}=\rho_{2} \cos \alpha_{2}, \quad q_{4}=\rho_{2} \sin \alpha_{2}
$$

and its canonical extension, $\left(Q_{1}, Q_{2}, Q_{3}, Q_{4}\right) \rightarrow\left(P_{1}, P_{2}, A_{1}, A_{2}\right)$

$$
P_{1}=\frac{q_{1} Q_{1}+q_{2} Q_{2}}{\sqrt{q_{1}^{2}+q_{2}^{2}}}, \quad A_{1}=q_{1} Q_{2}-q_{2} Q_{1}, \quad P_{2}=\frac{q_{3} Q_{3}+q_{4} Q_{4}}{\sqrt{q_{3}^{2}+q_{4}^{2}}}, \quad A_{2}=q_{3} Q_{4}-q_{4} Q_{3}
$$

Then, the Hamiltonian (1) in the new variables reads

$$
\mathcal{H}_{O}=\frac{1}{2}\left(P_{1}^{2}+P_{2}^{2}+\frac{A_{1}^{2}}{\rho_{1}^{2}}+\frac{A_{2}^{2}}{\rho_{2}^{2}}+\omega\left(\rho_{1}^{2}+\rho_{2}^{2}\right)+\frac{a}{\rho_{1}^{2}}+\frac{b}{\rho_{2}^{2}}\right)
$$

Note that $\alpha_{1}$ and $\alpha_{2}$ are cyclic, thus $A_{1}$ and $A_{2}$ are first integrals. Take into account that we will have to deal with the quadratures of

$$
\dot{\alpha}_{1}=\frac{\partial \mathcal{H}_{O}}{\partial A_{1}}=\frac{A_{1}}{\rho_{1}^{2}}, \quad \dot{\alpha}_{2}=\frac{\partial \mathcal{H}_{O}}{\partial A_{2}}=\frac{A_{2}}{\rho_{2}^{2}}
$$

after having integrated the rest of the system. 
Introducing the quantities

$$
k_{1}=A_{1}^{2}+a, \quad k_{2}=A_{2}^{2}+b,
$$

our Hamiltonian may be written as

$$
\mathcal{H}_{O}=\frac{1}{2}\left(P_{1}^{2}+P_{2}^{2}+\omega\left(\rho_{1}^{2}+\rho_{2}^{2}\right)+\frac{k_{1}}{\rho_{1}^{2}}+\frac{k_{2}}{\rho_{2}^{2}}\right),
$$

in other words, the two dimensional Smorodinsky-Winternitz system (see Evans 1990) in the variables $\left(\rho_{1}, \rho_{2}, P_{1}, P_{2}\right)$. Other authors call this Hamiltonian (9) a set of two non-interacting isotonic systems with the same frequency.

Apart from the constants of motion defined by the energies of each degree of freedom, it is well known that there is another integral of motion given by

$$
\mathcal{C}=\left(\rho_{1} P_{2}-\rho_{2} P_{1}\right)^{2}+k_{1} \frac{\rho_{2}^{2}}{\rho_{1}^{2}}+k_{2} \frac{\rho_{1}^{2}}{\rho_{2}^{2}} .
$$

Thus our Hamiltonian defines a superintegrable system, but we will not elaborate on this issue here. Just note that those systems, apart from being integrable in the LiouvilleArnold sense, they possess more constants of motion than degrees of freedom. Moreover, if the number $\mathrm{N}$ of independent constants takes the value $N=2 n-1$, then the system is called maximally superintegrable. Apart from the three well known classic cases, more recently other less simple models such as the Calogero-Moser, the Smorodinsky-Winternitz and the hyperbolic Calogero-Sutherland-Moser have been identified as superintegrable n-dimensional systems (López et al. 1999).

\subsection{The explicit solution}

The system is made separable in two subsystems of one degree of freedom defined by the Hamiltonian functions

$$
\mathcal{H}_{a}=\frac{1}{2}\left(P_{1}^{2}+\frac{A_{1}^{2}+a}{\rho_{1}^{2}}+\omega \rho_{1}^{2}\right), \quad \mathcal{H}_{b}=\frac{1}{2}\left(P_{2}^{2}+\frac{A_{2}^{2}+b}{\rho_{2}^{2}}+\omega \rho_{2}^{2}\right),
$$

such that

$$
\mathcal{H}_{O}=\mathcal{H}_{a}+\mathcal{H}_{b}
$$

We integrate the differential system defined by (6) immediately, following closely the approach given by Deprit (1991). It is based on a slight variation of the Hamilton-Jacobi scheme that he calls Delaunay transformation. Introducing

$$
\tilde{Q}=2 \mathcal{H}_{a}-\omega \rho_{1}^{2}-\frac{A_{1}^{2}+a}{\rho_{1}^{2}},
$$

and the quantities $a_{1}$ and $b_{1}$

$$
a_{1}+b_{1}=\sqrt{2\left(\frac{\mathcal{H}_{a}}{\omega}+\sqrt{\frac{A_{1}^{2}+a}{\omega}}\right)}, \quad a_{1}-b_{1}=\sqrt{2\left(\frac{\mathcal{H}_{a}}{\omega}-\sqrt{\left.\frac{A_{1}^{2}+a}{\omega}\right)}\right.},
$$


Fig. 1 Generic solution of the 4-D Hamiltonian (1). Left projection $\left(q_{1}, q_{2}\right)$, right $\left(q_{3}, q_{4}\right)$.
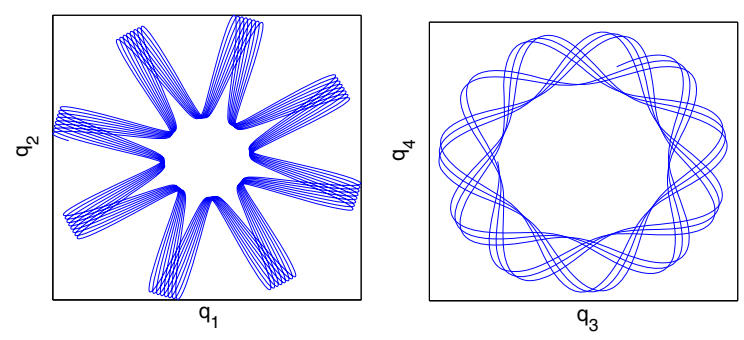

we may write

$$
\tilde{Q}=\frac{\omega}{\rho_{1}^{2}}\left(a_{1}^{2}-\rho_{1}^{2}\right)\left(\rho_{1}^{2}-b_{1}^{2}\right)
$$

We see that the equation $\tilde{Q}=0$ has real roots when $\mathcal{H}_{a} \geq \sqrt{\omega\left(A_{1}^{2}+a\right)}$. The system defined by the Hamiltonian $\mathcal{H}_{a}$ is reduced to

$$
\frac{d \rho_{1}}{d \tau}=P_{1}=\sqrt{\tilde{Q}}, \quad \frac{d \alpha_{1}}{d \tau}=\frac{A_{1}}{\rho_{1}^{2}},
$$

i.e., to two quadratures whose integrals are

$$
\rho_{1}(\tau)=\sqrt{a_{1}^{2} \sin ^{2} \sqrt{\omega} \tau+b_{1}^{2} \cos ^{2} \sqrt{\omega} \tau}, \quad \alpha_{1}(\tau)=\frac{A_{1}}{a_{1} b_{1} \sqrt{\omega}} \tan ^{-1}\left(\frac{a_{1}}{b_{1}} \tan \sqrt{\omega} \tau\right) .
$$

Note that in the literature this solution may be also obtained as a particular case of the so-called Pinney-Ermakov equation (see Cariñena et al. 2007).

Analogous computations for the Hamiltonian $\mathcal{H}_{b}$ in Eq. 11, with quantities $a_{2}$ and $b_{2}$ given by

$$
a_{2}+b_{2}=\sqrt{2\left(\frac{\mathcal{H}_{b}}{\omega}+\sqrt{\frac{A_{2}^{2}+b}{\omega}}\right)}, \quad a_{2}-b_{2}=\sqrt{2\left(\frac{\mathcal{H}_{b}}{\omega}-\sqrt{\frac{A_{2}^{2}+b}{\omega}}\right)}
$$

produce

$$
\rho_{2}(\tau)=\sqrt{a_{2}^{2} \sin ^{2} \sqrt{\omega} \tau+b_{2}^{2} \cos ^{2} \sqrt{\omega} \tau}, \quad \alpha_{2}(\tau)=\frac{A_{2}}{a_{2} b_{2} \sqrt{\omega}} \tan ^{-1}\left(\frac{a_{2}}{b_{2}} \tan \sqrt{\omega} \tau\right) .
$$

The equilibrium points of the reduced systems are the zeros of the potential functions, i.e. $\rho_{i}^{4}=k_{i} / \omega,(i=1,2)$, which correspond to circular orbits with minimal energies $\mathcal{H}_{a}=$ $\sqrt{\omega k_{1}}$ and $\left.\mathcal{H}_{b}=\sqrt{\omega k_{2}}\right)$.

In each of the two separable subsystems the generic solution is a rosetta type-orbit, periodic or not, see Fig. 1. In particular, circular orbits appear as a limit case of the rosetta, but considering here as another orbit type due to their simplicity.

Therefore, it can be stated that the generic solution of the Hamiltonian system (1) is a pair of non-periodic rosetta orbits. Three different family classes are obtained: two rosetta orbits, one circular and one rosetta, or two circular orbits.

As our Hamiltonian system is separable in two subsystems in $\left(\rho_{1}, \alpha_{2}\right)$ and $\left(\rho_{2}, \alpha_{2}\right)$, related by means of the energy of the complete system, we may take advantage of this property to obtain periodic orbits of the complete system, as we will see in the next section. 


\section{Analysis of periodic orbits}

In this section we present an analysis of the orbits of the 4-D dynamical system, studying the resonances needed to obtain a periodic orbit. From the solutions given in Sect. 2.2 note that the radii $\rho_{1}$ and $\rho_{2}$ are periodic quantities with period $T_{\rho}=\pi / \sqrt{\omega}$. The angular quantities $\alpha_{1}$ and $\alpha_{2}$ are also periodic with periods $T_{\alpha_{1}}$ and $T_{\alpha_{2}}$, respectively. Nevertheless, note that in general

$$
\alpha_{i}\left(n T_{\rho}\right)=n \pi \frac{A_{i}}{a_{i} b_{i} \sqrt{\omega}} \neq 2 m \pi, \quad(i=1,2),
$$

where $m, n$, are integers.

Therefore, we will only find periodic solutions of the 4-D Hamiltonian (1) when the two resonance conditions

$$
a_{1} b_{1} \sqrt{\omega} / A_{1} \in \mathbb{Q}, \quad a_{2} b_{2} \sqrt{\omega} / A_{2} \in \mathbb{Q},
$$

are satisfied.

In case of a pair of circular orbits only one commensurability relation is needed. By means of Eqs. 13 and 14 we can rewrite $a_{1} b_{1} \sqrt{\omega} / A_{1}=\left(\sqrt{A_{1}^{2}+a} / \sqrt{\omega}\right) \sqrt{\omega} / A_{1}$, and so the resonance condition for circular-circular orbits is

$$
m A_{2} \sqrt{A_{1}^{2}+a}=n A_{1} \sqrt{A_{2}^{2}+b}, \quad m, n \in \mathbb{Z},
$$

while Eq. 15 must be satisfied for all other rosetta-circular or rosetta-rosetta pairs of orbits.

\subsection{Computing periodic solutions}

In order to search for periodic orbits, we take fixed values of the parameters $a, b$ and total energy $\mathcal{H}_{0}\left(=\mathcal{H}_{a}+\mathcal{H}_{b}\right)$, and compute closed trajectories for each subsystems $\mathcal{H}_{a}, \mathcal{H}_{b}$ separately.

The simplest case is to consider circular orbits, whose radii are $\rho_{i}=\left(k_{i} / \omega\right)^{1 / 4}$. We illustrate them in Fig. 2 by plotting the phase portrait of any of these subsystems, $\mathcal{H}_{a}$ or $\mathcal{H}_{b}$, for some given value of the parameters. The phase portrait represents multiple curves corresponding to different initial conditions in the same phase plane, and provides useful information about the system's trajectories. Indeed Fig. 2 depicts a stable steady state in a phase space, where the fixed point corresponds to a circular orbit. The existence of this orbit in one of the separable subsystems (11) results in a 3-dimensional family of invariant tori, or a 2-tori out of a pair of circular orbits. Note that these tori may be seen as foliations of the Lagrangian tori associated to the system (1) when we take into account the existence of the reduced integral (10) of the reduced system (9) which has all orbits periodic. For more details see Balsas et al. (2009).

In order to compute the periodic orbit for given values of the parameters of the problem $a$ and $b$, we consider a certain value of the energy of the complete system and we take some initial conditions $\left(q_{1}, q_{2}\right)$ of a circular orbit for the system $\mathcal{H}_{a}$. The energy of this orbit imposes a value for $\mathcal{H}_{b}$, leading to obtain a second circular orbit $\left(q_{3}, q_{4}\right)$ which is related to the first one by means of the energy.

The largest circular orbit $\left(q_{1}, q_{2}\right)$ implies a greater value of the energy $\mathcal{H}_{a}$, leading to a smaller $\mathcal{H}_{b}$ value for the circular orbit in the $\left(q_{3}, q_{4}\right)$ plane. These orbits do not produce in general a 4-D periodic orbit. We must choose the appropriated initial conditions of both 
Fig. 2 Phase portrait of a typical subsystem with Hamiltonian $\mathcal{H}_{a}$ or $\mathcal{H}_{b}$, where a center is depicted

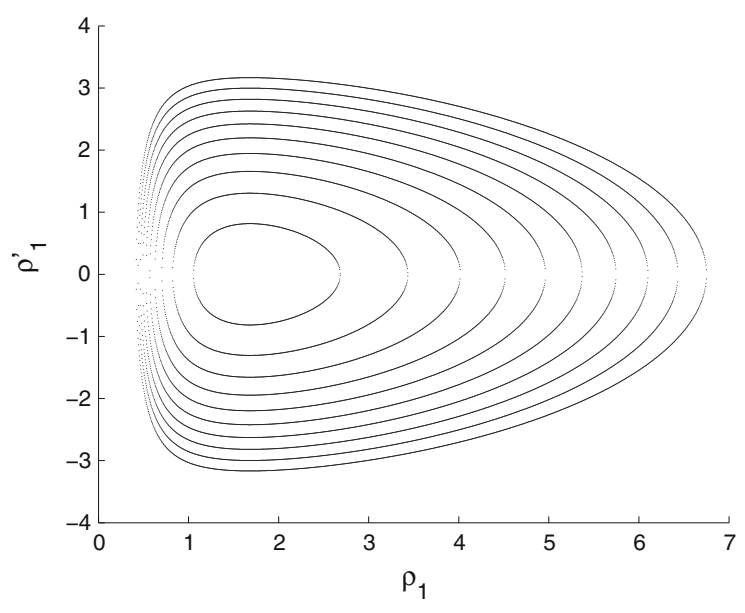

Fig. 3 Example of 4-D periodic orbit out of two rosetta 2-D periodic orbits. Left $\left(q_{1}, q_{2}\right)$, right $\left(q_{3}, q_{4}\right)$
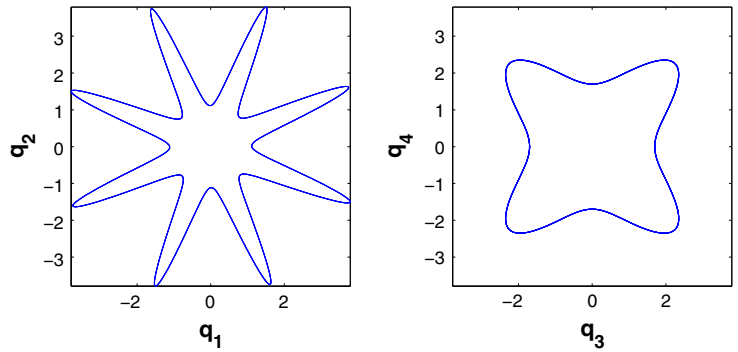

orbits so that the resonance condition (16) is satisfied, and so we can obtain a periodic orbit in the complete system with period the product of the period of the orbits $\left(q_{1}, q_{2}\right)$ and $\left(q_{3}, q_{4}\right)$.

Remind that the commensurability conditions (15) apply to rosetta-type orbits, and therefore this same procedure applies for all orbits type. Figure 3 depicts another example of a 4-D periodic orbit whose projections $\left(q_{1}, q_{2}\right)$ and $\left(q_{3}, q_{4}\right)$ are a pair of rosetta orbits.

Hence, thanks to the separability property of the 4-D Hamiltonian system, we have a simple way of computing 4-D periodic orbits of the complete problem out of 2-D orbits of its subsystems satisfying the periodicity relations.

\section{Hartmann and isotropic potentials. Families of periodic orbits}

In this section are presented two families of three dimensional systems involving ring shaped potentials, the Hartmann and the Isotropic systems, which are reductions of the initial Hamiltonian (1). We also describe some particular periodic orbits of both potentials and the generation of initial conditions of 4-D orbits of (1) through the computation of periodic orbits of the 3-D reduced systems.

\subsection{The oscillator and the generalized Hartmann potentials}

We show first the relation of the Hamiltonian system (1) and the generalized Hartmann potentials $V_{1}$ defined by Eq. 2 . In order to do that we will use the transformation $(r, \phi, \lambda, \psi) \rightarrow$ $\left(q_{1}, q_{2}, q_{3}, q_{4}\right)$ given by 


$$
\begin{aligned}
& q_{1}=\sqrt{r} \sin \frac{\phi}{2} \cos \frac{\lambda-\psi}{2}, \quad q_{3}=\sqrt{r} \cos \frac{\phi}{2} \sin \frac{\lambda+\psi}{2}, \\
& q_{2}=\sqrt{r} \sin \frac{\phi}{2} \sin \frac{\lambda-\psi}{2}, \quad q_{4}=\sqrt{r} \cos \frac{\phi}{2} \cos \frac{\lambda+\psi}{2},
\end{aligned}
$$

with $(r, \phi, \lambda, \psi) \in R^{+} \times(0, \pi) \times[0,2 \pi] \times\left(-\frac{\pi}{2}, \frac{\pi}{2}\right)$, and whose Jacobian is $-r \sin \phi / 8$. Later on we will need the inverse transformation written as

$$
\begin{aligned}
& r=q_{1}^{2}+q_{2}^{2}+q_{3}^{2}+q_{4}^{2}, \\
& \sin \phi=\frac{2 \sqrt{\left(q_{1}^{2}+q_{2}^{2}\right)\left(q_{3}^{2}+q_{4}^{2}\right)}}{q_{1}^{2}+q_{2}^{2}+q_{3}^{2}+q_{4}^{2}}, \quad \cos \phi=\frac{q_{3}^{2}+q_{4}^{2}-q_{1}^{2}-q_{2}^{2}}{q_{1}^{2}+q_{2}^{2}+q_{3}^{2}+q_{4}^{2}}, \\
& \sin \lambda=\frac{q_{1} q_{3}+q_{2} q_{4}}{\sqrt{\left(q_{1}^{2}+q_{2}^{2}\right)\left(q_{3}^{2}+q_{4}^{2}\right)}}, \quad \cos \lambda=\frac{q_{1} q_{4}-q_{2} q_{3}}{\sqrt{\left(q_{1}^{2}+q_{2}^{2}\right)\left(q_{3}^{2}+q_{4}^{2}\right)}}, \\
& \sin \psi=\frac{q_{1} q_{3}-q_{2} q_{4}}{\sqrt{\left(q_{1}^{2}+q_{2}^{2}\right)\left(q_{3}^{2}+q_{4}^{2}\right)}}, \quad \cos \psi=\frac{q_{1} q_{4}+q_{2} q_{3}}{\sqrt{\left(q_{1}^{2}+q_{2}^{2}\right)\left(q_{3}^{2}+q_{4}^{2}\right)}} .
\end{aligned}
$$

These variables are well known in the literature. See the works of Kibler and Négadi, Ikeda and Miyachi (1971), and the classical physics book of Synge (1960). In Cornish (1984), we find a reference to the work of Barut et al. (1979), where the variables are introduced starting from the transformation $\left(\zeta_{A}, \zeta_{B}\right) \rightarrow(x, y, z, \sigma)$

$$
x+i y=2 \zeta_{A} \bar{\zeta}_{B}, \quad z=\zeta_{A} \bar{\zeta}_{A}-\zeta_{B} \bar{\zeta}_{B}, \quad \sigma=\arg \zeta_{A} \zeta_{B},
$$

where $\zeta_{A}$ y $\zeta_{B}$ are two complex variables. We find them also in Stiefel and Scheifele (1971), although no further use of them is done. As these variables are related to Euler angles of rotation, we propose to denote them as Euler projective variables.

The canonical extension associated to the transformation (17) is obtained as a Mathieu transformation, satisfying $\sum Q_{i} d q_{i}=R d r+\Phi d \phi+\Lambda d \lambda+\Psi d \psi$. Therefore, the relations among the momenta are given by

$$
\begin{aligned}
R & =\frac{1}{2 \sum q_{i}^{2}}\left(q_{1} Q_{1}+q_{2} Q_{2}+q_{3} Q_{3}+q_{4} Q_{4}\right), \\
\Phi & =\frac{\left(q_{1} Q_{1}+q_{2} Q_{2}\right)\left(q_{3}^{2}+q_{4}^{2}\right)-\left(q_{3} Q_{3}+q_{4} Q_{4}\right)\left(q_{1}^{2}+q_{2}^{2}\right)}{2 \sqrt{\left(q_{1}^{2}+q_{2}^{2}\right)\left(q_{3}^{2}+q_{4}^{2}\right)}} \\
\Lambda & =\frac{1}{2}\left(-q_{2} Q_{1}+q_{1} Q_{2}+q_{4} Q_{3}-q_{3} Q_{4}\right), \\
\Psi & =\frac{1}{2}\left(q_{2} Q_{1}-q_{1} Q_{2}+q_{4} Q_{3}-q_{3} Q_{4}\right) .
\end{aligned}
$$

The Hamiltonian (1) in the new variables may be written as

$$
\mathcal{H}=4 r\left[\frac{\omega}{8}+\frac{1}{2}\left(R^{2}+\frac{\Phi^{2}}{r^{2}}+\frac{\Lambda^{2}}{r^{2} \sin ^{2} \phi}\right)+\frac{\Psi^{2}-2 \Lambda \Psi \cos \phi}{2 r^{2} \sin ^{2} \phi}+\frac{c+d \cos \phi}{2 r^{2} \sin ^{2} \phi}\right],
$$

where

$$
c=\frac{a+b}{2}, \quad d=\frac{a-b}{2} .
$$


Note that $\lambda$ and $\psi$ are cyclic variables, and so $\Lambda$ and $\Psi$ are first integrals. In other words the differential systems is described by

$$
\frac{d r}{d \tau}=\frac{\partial \mathcal{H}}{\partial R}, \quad \frac{d \phi}{d \tau}=\frac{\partial \mathcal{H}}{\partial \Phi}, \quad \frac{d R}{d \tau}=-\frac{\partial \mathcal{H}}{\partial r}, \quad \frac{d \Phi}{d \tau}=-\frac{\partial \mathcal{H}}{\partial \phi},
$$

and two quadratures $\lambda=\int(\partial \mathcal{H} / \partial \Lambda) d \tau$ and $\psi=\int(\partial \mathcal{H} / \partial \Psi) d \tau$.

Using Poincaré notation and introducing a change of independent variable $\tau \rightarrow s$ given by $d \tau=4 r d s$, the Hamiltonian $\mathcal{K}=(\mathcal{H}-h) /(4 r)$ takes the expression

$$
\mathcal{K}=\frac{\omega}{8}+\frac{1}{2}\left(R^{2}+\frac{\Phi^{2}}{r^{2}}+\frac{\Lambda^{2}}{r^{2} \sin ^{2} \phi}\right)+\frac{\Psi^{2}-2 \Lambda \Psi \cos \phi}{2 r^{2} \sin ^{2} \phi}+\frac{c+d \cos \phi}{2 r^{2} \sin ^{2} \phi}-\frac{h}{4 r},
$$

where $h$ is a fixed value of the Hamiltonian $\mathcal{H}$ for chosen initial conditions, and the flow is defined now on the manifold $\mathcal{K}=0$. We prefer to use a slightly different form, and we consider the Hamiltonian

$$
\tilde{\mathcal{K}}=\frac{1}{2}\left(R^{2}+\frac{\Phi^{2}}{r^{2}}+\frac{\Lambda^{2}}{r^{2} \sin ^{2} \phi}\right)-\frac{h}{4 r}+\frac{\left(\Psi^{2}+c\right) / 2}{r^{2} \sin ^{2} \phi}+\frac{(d / 2-\Lambda \Psi) \cos \phi}{r^{2} \sin ^{2} \phi}
$$

in the manifold $\tilde{\mathcal{K}}=-\frac{\omega}{8}$.

Notice that this system has two cyclic variables $\lambda$ and $\psi$. Therefore, denoting

$$
\mathcal{K}^{*}=\frac{1}{2}\left(R^{2}+\frac{\Phi^{2}}{r^{2}}+\frac{\Lambda^{2}}{r^{2} \sin ^{2} \phi}\right)-\frac{h}{4 r}
$$

the differential system defined by (22) is given by

$$
\begin{aligned}
& \frac{d r}{d s}=\frac{\partial \tilde{\mathcal{K}}}{\partial R}=R, \\
& \frac{d \phi}{d s}=\frac{\partial \tilde{\mathcal{K}}}{\partial \Phi}=\frac{\Phi}{r^{2}}, \\
& \frac{d R}{d s}=-\frac{\partial \tilde{\mathcal{K}}}{\partial r}=-\frac{\partial \mathcal{K}^{*}}{\partial r}+\frac{\Psi^{2}+c+(d-2 \Lambda \Psi) \cos \phi}{r^{3} \sin ^{2} \phi}, \\
& \frac{d \Phi}{d s}=-\frac{\partial \tilde{\mathcal{K}}}{\partial \phi}=-\frac{\partial \mathcal{K}^{*}}{\partial \phi}-\frac{\left(\Psi^{2}+c\right) / 2}{r^{2}} \frac{\partial}{\partial \phi}\left(\frac{1}{\sin ^{2} \phi}\right)-\frac{(d / 2-\Lambda \Psi)}{r^{2}} \frac{\partial}{\partial \phi}\left(\frac{\cos \phi}{\sin ^{2} \phi}\right),
\end{aligned}
$$

and two quadratures

$$
\begin{aligned}
& \lambda=\int \frac{\partial \tilde{\mathcal{K}}}{\partial \Lambda} d s=\int \frac{\Lambda-\Psi \cos \phi}{r^{2} \sin ^{2} \phi} d s, \\
& \psi=\int \frac{\partial \tilde{\mathcal{K}}}{\partial \Psi} d s=\int \frac{\Psi-\Lambda \cos \phi}{r^{2} \sin ^{2} \phi} d s .
\end{aligned}
$$

If we consider now the differential system defined by the Hamiltonian function with potential $V_{1}$ in spherical variables $(r, \phi, \lambda)$,

$$
x_{1}=r \sin \phi \cos \lambda, \quad x_{2}=r \sin \phi \sin \lambda, \quad x_{3}=r \cos \phi,
$$

and their momenta $(R, \Phi, \Lambda)$, we will have

$$
\mathcal{H}_{1}=\mathcal{H}_{0}+\frac{P+Q \cos \phi}{r^{2} \sin ^{2} \phi} \text { where } \mathcal{H}_{0}=\frac{1}{2}\left(R^{2}+\frac{\Phi^{2}}{r^{2}}+\frac{\Lambda^{2}}{r^{2} \sin ^{2} \phi}\right)-\frac{\mu}{r}
$$


and the differential system will be written as

$$
\begin{aligned}
& \frac{d r}{d s}=\frac{\partial \mathcal{H}_{1}}{\partial R}=R, \\
& \frac{d \phi}{d s}=\frac{\partial \mathcal{H}_{1}}{\partial \Phi}=\frac{\Phi}{r^{2}}, \\
& \frac{d R}{d s}=-\frac{\partial \mathcal{H}_{1}}{\partial r}=-\frac{\partial \mathcal{H}_{0}}{\partial r}+\frac{P+Q \cos \phi}{r^{3} \sin ^{2} \phi}, \\
& \frac{d \Phi}{d s}=-\frac{\partial \mathcal{H}_{1}}{\partial \phi}=-\frac{\partial \mathcal{H}_{0}}{\partial \phi}-\frac{P}{r^{2}} \frac{\partial}{\partial \phi}\left(\frac{1}{\sin ^{2} \phi}\right)-\frac{Q}{r^{2}} \frac{\partial}{\partial \phi}\left(\frac{\cos \phi}{\sin ^{2} \phi}\right),
\end{aligned}
$$

and the quadrature

$$
\lambda=\int \frac{\partial \mathcal{H}_{1}}{\partial \Lambda} d s=\int \frac{\Lambda}{r^{2} \sin ^{2} \phi} d s .
$$

We check that these last equations coincide with Eqs. 23 and 24 when we restrict to the manifold $\Psi=0$, taking $h=4 \mu, c=2 P, d=2 Q$, and we identify the variable $s$ with the physical time $t$. Moreover, in this case the quadrature (25) takes the form

$$
\psi=\int \frac{\partial \tilde{\mathcal{K}}}{\partial \Psi} d s=-\int \frac{\Lambda \cos \phi}{r^{2} \sin ^{2} \phi} d s .
$$

Thus, we have shown that the dynamics of the oscillator defined by the Hamiltonian function (1) includes the family of the generalized Hartmann potentials. Note that we may interchange the role played by $\Lambda$ and $\Psi$. Further, when we restrict to the Hartmann case $(Q=0)$, we may simplify the 4-D potential reducing it to only one parameter $a=b$.

\subsection{Relation with generalized 3-D isotropic potentials}

There is still another family of potentials related to our system (1), the isotropis potential $V_{2}$, defined by Eq. 3. Let us consider now the transformation

$$
q_{1}=r \cos \alpha \cos \beta, \quad q_{2}=r \cos \alpha \sin \beta, \quad q_{3}=r \sin \alpha \cos \gamma, \quad q_{4}=r \sin \alpha \sin \gamma,
$$

with Jacobian $-r^{3} \sin 2 \alpha / 2$ i.e., $\alpha \in(0, \pi / 2) \bigcup(\pi / 2, \pi)$. This transformation was used by Kibler and Negali (1984a).

The associated canonical extension $(q, Q) \rightarrow(r, \alpha, \beta, \gamma, R, A, B, C)$ reads

$$
\begin{aligned}
R & =\frac{1}{\sqrt{\sum q_{i}^{2}}}\left(q_{1} Q_{1}+q_{2} Q_{2}+q_{3} Q_{3}+q_{4} Q_{4}\right), \\
A & =\frac{\left(q_{3} Q_{3}+q_{4} Q_{4}\right)\left(q_{1}^{2}+q_{2}^{2}\right)-\left(q_{1} Q_{1}+q_{2} Q_{2}\right)\left(q_{3}^{2}+q_{4}^{2}\right)}{\sqrt{\left(q_{1}^{2}+q_{2}^{2}\right)\left(q_{3}^{2}+q_{4}^{2}\right)}}, \\
B & =-q_{2} Q_{1}+q_{1} Q_{2}, \\
C & =-q_{4} Q_{3}+q_{3} Q_{4},
\end{aligned}
$$


while the inverse transformation, needed for the construction of the explicit transformation with the old variables, takes the form

$$
\begin{array}{rlrl}
r & =\sqrt{q_{1}^{2}+q_{2}^{2}+q_{3}^{2}+q_{4}^{2}}, & & \\
\cos \alpha & =\sqrt{\frac{q_{1}^{2}+q_{2}^{2}}{q_{1}^{2}+q_{2}^{2}+q_{3}^{2}+q_{4}^{2}},} & \sin \alpha=\sqrt{\frac{q_{3}^{2}+q_{4}^{2}}{q_{1}^{2}+q_{2}^{2}+q_{3}^{2}+q_{4}^{2}}} \\
\cos \beta & =\frac{q_{1}}{\sqrt{q_{1}^{2}+q_{2}^{2}}}, & \sin \beta & =\frac{q_{2}}{\sqrt{q_{1}^{2}+q_{2}^{2}}}, \\
\cos \gamma & =\frac{q_{3}}{\sqrt{q_{3}^{2}+q_{4}^{2}}}, & \sin \gamma & =\frac{q_{4}}{\sqrt{q_{3}^{2}+q_{4}^{2}}} .
\end{array}
$$

The Hamiltonian (1) in these variables is given by

$$
\mathcal{H}=\frac{1}{2}\left(R^{2}+\frac{A^{2}}{r^{2}}+\frac{C^{2}}{r^{2} \sin ^{2} \alpha}\right)+\frac{\omega}{2} r^{2}+\frac{B^{2}+a}{2 r^{2} \cos ^{2} \alpha}+\frac{b}{2 r^{2} \sin ^{2} \alpha} .
$$

Note that $\beta$ and $\gamma$ are cyclic, thus $B$ and $C$ are first integrals. In other words the differential systems is described by

$$
\frac{d r}{d \tau}=\frac{\partial \mathcal{H}}{\partial R}, \quad \frac{d \alpha}{d \tau}=\frac{\partial \mathcal{H}}{\partial A}, \quad \frac{d R}{d \tau}=-\frac{\partial \mathcal{H}}{\partial r}, \quad \frac{d A}{d \tau}=-\frac{\partial \mathcal{H}}{\partial \alpha}
$$

and two quadratures

$$
\beta=\int \frac{\partial \mathcal{H}}{\partial B} d \tau=\int \frac{B}{r^{2} \cos ^{2} \alpha} d \tau, \quad \gamma=\int \frac{\partial \mathcal{H}}{\partial C} d \tau=\int \frac{C}{r^{2} \sin ^{2} \alpha} d \tau .
$$

If we restrict to the subsystem defined by $(r, \alpha, \gamma, R, A, C)$, and we consider the transformation defined by

$$
x_{1}=r \sin \alpha \cos \gamma, \quad x_{2}=r \sin \alpha \sin \gamma, \quad x_{3}=r \cos \alpha,
$$

the defined system corresponds to the one given by the family of potentials $V_{2}$, choosing the constants as follows

$$
\omega=\Omega^{2}, \quad a=P-B^{2} \quad \text { y } \quad b=Q,
$$

and identifying $\tau$ with the physical time $t$.

Only when $B=-C$ the dynamics of the 4-D oscillator belongs to the manifold $q_{2} Q_{1}-q_{1} Q_{2}+q_{4} Q_{3}-q_{3} Q_{4}=0$. Notice that the 3-D isotropic oscillator is obtained either choosing $B=a=b=0$, or if $b=0$ and $a=-B^{2}$. Kibler and Winternitz (1990) studied the case $P=0$, and a similar analysis for the general case may be found in Kibler et al. (1996).

Finally, as it has been described along this section, differential systems with potentials $V_{1}$ and $V_{2}$ are related to our system (1). Therefore, once we get a 4-D periodic orbit of the system (1) we can apply the relations between the 4-D oscillator and the family of Hartmann and isotropic potentials, to obtain the corresponding 3-D periodic orbits. Figure 4 shows the orbits obtained for both potentials, when we apply the transformations to the initial condition of a particular 4-D periodic orbit. This 4-D orbit was depicted in Fig. 3. 

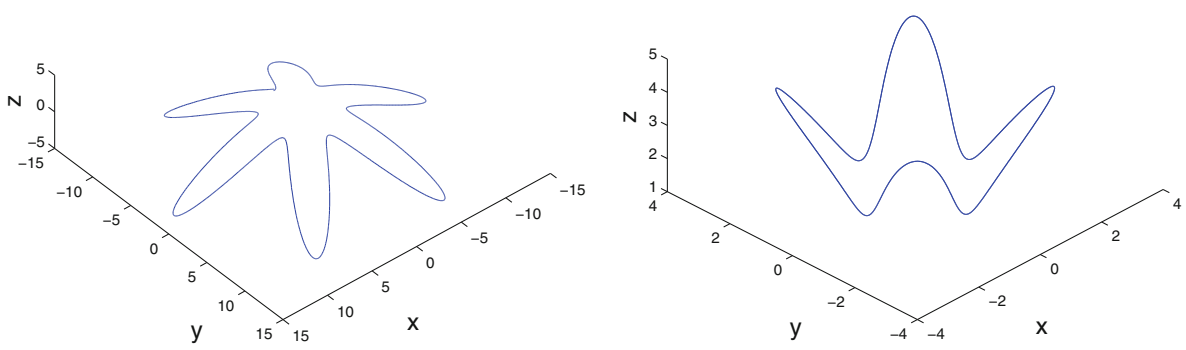

Fig. 4 Periodic orbits obtained through 4-D orbit shown in Fig. 3. Left Hartmann orbit in cylindric coordinates (26). Right Isotropic orbit in spherical coordinates (35).

\subsection{Some particular periodic orbits of the Hartmann and isotropic potentials}

Finally, we search for some particular periodic orbits of the Hartmann and Isotropic potentials, and their relation with the 4-D trajectories of the initial Hamiltonian (1).

In order to identify the special ring-shaped solutions, it is convenient to express the Hamiltonian defined by the potentials $V_{1}$ and $V_{2}$ in symplectic cylindric variables $(\lambda, \rho, z, \Lambda, P, Z)$,

$$
\mathcal{H}_{1}=\frac{1}{2}\left(P^{2}+Z^{2}+\frac{\Lambda^{2}}{\rho^{2}}\right)-\frac{\mu}{\sqrt{\rho^{2}+z^{2}}}+\frac{\tilde{P}}{\rho^{2}}+\frac{Q z}{\rho^{2} \sqrt{\rho^{2}+z^{2}}},
$$

and

$$
\mathcal{H}_{2}=\frac{1}{2}\left(P^{2}+Z^{2}+\frac{\Lambda^{2}}{\rho^{2}}\right)+\frac{\Omega^{2}}{2}\left(\rho^{2}+z^{2}\right)+\frac{\tilde{P}}{2 z^{2}}+\frac{Q}{2 \rho^{2}},
$$

where we have denoted the parameter $P \equiv \tilde{P}$ to avoid confusion with the cylindric momentum $P$. Note that both potentials are axially symmetric with the cyclic variable $\lambda=\int\left(\Lambda / \rho^{2}\right) d t$.

Then, we will focus on the characterization of three type of possible periodic solutions, namely the ones defined by $\rho$ constant, $z$ constant, or both.

(i) $(\rho, z)$ constant

Related to $V_{2}$ potentials, the family of orbits is composed of circles around the $O z$ axis and is given by

$$
\dot{P}=\frac{\partial \mathcal{H}_{2}}{\partial \rho}=\frac{\Lambda^{2}}{\rho^{3}}-\Omega^{2} \rho+\frac{Q}{\rho^{3}}=0, \quad \dot{Z}=\frac{\partial \mathcal{H}_{2}}{\partial z}=-\Omega^{2} z+\frac{\tilde{P}}{z^{3}}=0 .
$$

The solution is $\left(\rho_{0}, z_{0}\right)=\left(\sqrt[4]{\left(\Lambda^{2}+Q\right) / \Omega^{2}}, \sqrt[4]{\tilde{P} / \Omega^{2}}\right)$. Now $\lambda=\int\left(\Lambda / \rho_{0}^{2}\right) d t$, when $\Lambda=0$ it reduces to a circle of equilibria.

Looking for similar type of solutions in $V_{1}$ potentials we find

$$
\begin{aligned}
& \dot{P}=\frac{\partial \mathcal{H}_{1}}{\partial \rho}=\frac{\Lambda^{2}+2 \tilde{P}}{\rho^{3}}-\frac{\mu \rho}{\left(\rho^{2}+z^{2}\right)^{3 / 2}}+\frac{Q z\left(3 \rho^{2}+z^{2}\right)}{\rho^{3}\left(\rho^{2}+z^{2}\right)^{3 / 2}}=0, \\
& \dot{Z}=\frac{\partial \mathcal{H}_{1}}{\partial z}=\frac{Q-\mu z}{\left(\rho^{2}+z^{2}\right)^{3 / 2}}=0 .
\end{aligned}
$$


Then, solutions $\left(\rho_{0}, z_{0}\right)$ are $z_{0}=Q / \mu$ and $\rho_{0}=\sqrt{x} / \mu$, where $x$ is a positive root of the polynomial equation which may be readily obtained from Eq. 39 .

\section{(ii) $\rho$ constant}

A second special type of solutions are those satisfying that $\rho$ is constant i.e. trajectories on a cylinder. Searching for them in the isotropic family $V_{2}$ we get $\rho_{0}=\sqrt[4]{\left(\Lambda^{2}+Q\right) / \Omega^{2}}$ and the differential equation

$$
\ddot{z}=-\Omega^{2} z+\frac{\tilde{P}}{z^{3}} .
$$

By means of its Hamiltonian function Eq. 37, an alternative to Eq. 41 can be written as

$$
\dot{z}=Z=\sqrt{D-\Omega^{2} z^{2}-\frac{\tilde{P}}{z^{2}}},
$$

where $D=2\left(\mathcal{H}_{2}-\Omega \sqrt{\Lambda^{2}+Q}\right)$. Then, introducing the variable $u=z^{2}$, and differentiating we obtain

$$
\int \frac{d u}{\sqrt{-\Omega^{2} u^{2}+D u-\tilde{P}}}=2\left(t-t_{0}\right),
$$

i.e. we get a solution that oscillates on the cylinder in the circumferences $\rho_{0}$.

Likewise, trying to identify this type of periodic solutions in the Hartmann family, we find that the answer is negative.

(iii) $z$ constant

A third special type of orbits are planar trajectories with $z$ constant. Again, for the isotropic family they are easily identified in the plane $z_{0}=\sqrt[4]{\tilde{P} / \Omega^{2}}$ with $\rho$ satisfying

$$
\ddot{\rho}=-\Omega^{2} \rho+\frac{\Lambda^{2}+Q}{\rho^{3}} .
$$

With respect to the possible solution for the Hartmann family, in the plane $z_{0}=Q / \mu$, the differential equation to be studied is reduced to the analysis of the elliptic integral

$$
-\int \frac{d v}{v^{2} \sqrt{-4 \mu Q^{2} v^{3}-\left(2 \mathcal{H}_{1} Q^{2}+\mu^{2}\left(2 \tilde{P}+\Lambda^{2}\right)\right) v^{2}+2 \mu^{3} v+2 \mu^{2} \mathcal{H}_{1}}}=\frac{1}{\mu}\left(t-t_{0}\right),
$$

where we have made use of the intermediary variable $v$ related to $\rho$ by $v^{2}=\left(\rho^{2}+z_{0}^{2}\right)^{-1}$.

\subsection{From three to four dimensions}

At last, note that any of these particular periodic orbits or a generic one can be used in order to generate initial conditions of a 4-D periodic orbit of the Hamiltonian system (1). Here is briefly described the procedure performed in the case of Isotropic potentials. Analogous treatment has to be followed for the Hartmann potential family. 

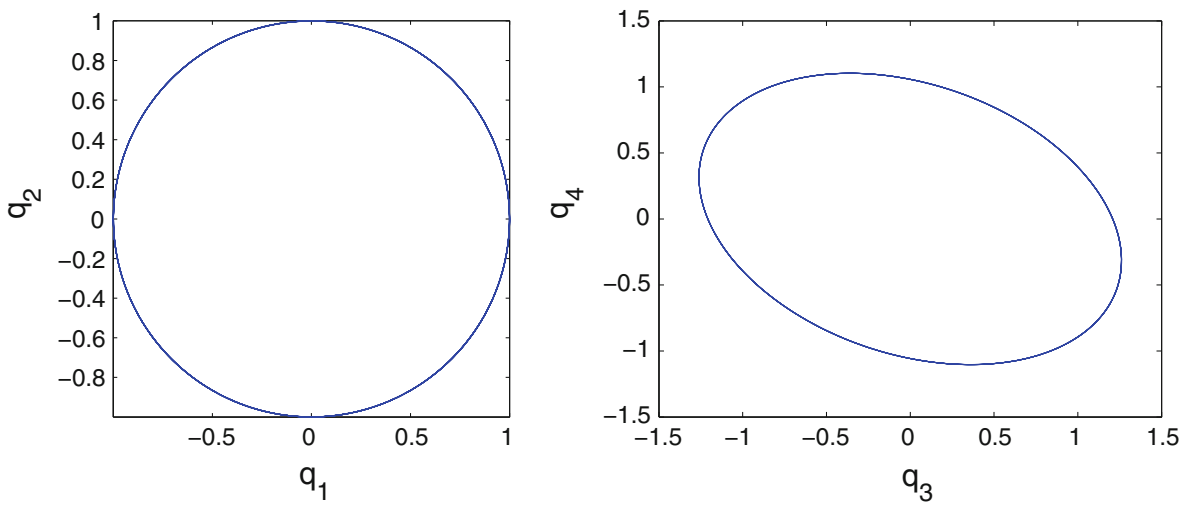

Fig. 5 Corresponding 4-D trajectory of $V_{2}$ orbit with $z$ constant

Once we have a set of initial conditions of a periodic orbit for the potential $V_{2}$ with a given value of the parameters $P$ and $Q$, the inverse transformation of the relation between the 4-D oscillator and the 3-D isotropic potential,

$$
x=r \sin \alpha \cos \gamma, \quad y=r \sin \alpha \sin \gamma, \quad x=r \cos \alpha,
$$

described in Sect. 4.2, allows us to obtain the spherical variables $(r, \alpha, \gamma)$ and their derivatives. By means of the formulas detailed in that section, Eqs. 30 and 31, the coordinates and moments $\left(q_{3}, q_{4}, Q_{3}, Q_{4}\right)$ are derived straightaway.

Our goal is to obtain a 4-D periodic orbit, thence it is mandatory to impose the commensurability conditions (15) for both separable systems $\left(q_{1}, q_{2}, Q_{1}, Q_{2}\right)$ and $\left(q_{3}, q_{4}, Q_{3}, Q_{4}\right)$,

$$
\frac{\sqrt{A_{1}^{2}+a}}{A_{1}}=\frac{m_{1}}{n_{1}}, \quad \frac{\sqrt{A_{2}^{2}+b}}{A_{2}}=\frac{m_{2}}{n_{2}},
$$

where we have been set the parameter of the initial Hamiltonian (1), $\omega=1$, for the sake of simplicity. Note that each set of initial conditions in cartesian coordinates determines a value of $A_{1}=q_{1} Q_{2}-q_{2} Q_{1}$ and $A_{2}=q_{3} Q_{4}-q_{4} Q_{3}$.

On the other hand, as we are jumping from 3-D to 4-D motion, the variables $\beta, B$ in Eqs. 30 and 31 are undetermined, from which it follows that $\left(q_{1}, q_{2}, Q_{1}, Q_{2}\right)$ joint with the parameter $a$, will be determined only taking into account the fulfillment of the commensurability relation. In this way we obtain a complete set of variables and parameters needed to integrate the 4-D Hamiltonian Eq. 1 providing a 4-D periodic orbit.

Next we present two figures depicting two examples related to the previous study. In Fig. 5 we represent the 4-D orbit obtained through one of the particular orbits of the $V_{2}$ family, with $z$ constant, above described. Figure 6 shows the 4 -D orbit originated out of a generic periodic orbit in which none of $(\rho, z)$ remain constant.

\section{Conclusion and future work}

We have established the relation of two families of ring-shaped type systems with a generalized integrable 4-D isotropic oscillator. This allows a unified treatment which is of interest both in quantum and classical studies. In particular conditions for periodic families have been 

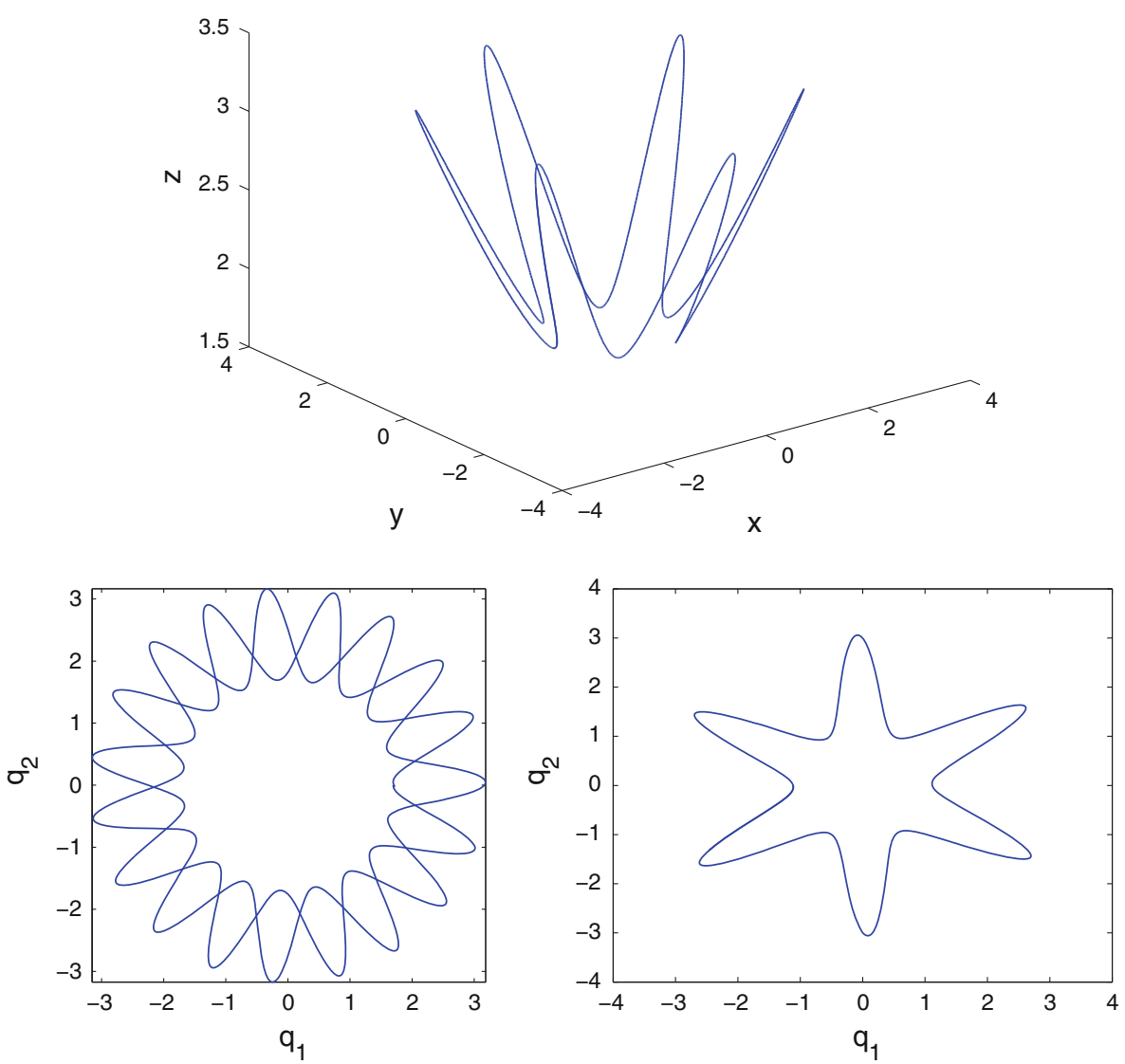

Fig. $6 \quad V_{2}$ generic periodic orbit (top) used to generate a 4-D orbit (bottom)

established. Topological aspects of this system, including its foliation by means of the first integrals will be presented in another paper (see Balsas et al.).

Acknowledgments We appreciate comments and suggestions from Dr. Elipe who improved the clarity and structure of the paper. We thank partial support from projects ESP-2005-07107 and MTM 2009-10767 of the Ministry of Technology and Science of Spain, and a grant from Fundación Séneca, of the Autonomous Region of Murcia.

\section{References}

Balsas, C., Ferrer, S., Jiménez, E., Vera, J.A.: Foliations of a generalized 4-D isotropic oscillator, submitted to International Journal of Bifurcation and Chaos (2009)

Barut, A.O., Schneider, C.K., Wilson, R.: Solution of a three-body problem in one dimension. J. Math. Phys. 20, 2244-2256 (1979)

Bhaduri, R.J., Sakhr, J., Sprung, D., Dutt, R., Suzuki A.: Shape invariant potentials in susy quantum mechanics and periodic orbit theory, arXiv:quant-ph/0410041 v2 (2005)

Calogero, F.: Solution of a three-body problem in one dimension. J. Math. Phys. 10, 2191-2196 (1969) 
Cariñena, J.F., Rañada, M.F., Santander, M.: A super-integrable two-dimensional non-linear oscillator with an exactly solvable quantum analog. In: Symmetry, integrability and geometry: methods and applications, SIGMA 3, 030, 23 p. (2007)

Chen, C.Y., Liu, C.L., Sun, D.S.: The normalized wavefunctions of the Hartmann potential and explicit expressions for their radial average values. Phys. Lett. A. 305, 341-348 (2002)

Cornish, F.H.: The hydrogen atom and the four-dimensional harmonic oscillator. J. Phys. A Math. Gen. 17, 323-327 (1984)

Deprit, A.: The Lissajous Transformation: I. Basics. Celest. Mech. Dyn. Astron. 51, 201-225 (1991)

Deprit, A., Henrard, J.: Natural families of periodic orbits. Astron. J. 72, 158-172 (1967)

Evans, N.W.: Superintegrability of the Smorodinsky-Winternitz system. Phys. Lett. 147, 483-486 (1990)

Fassò, F.: Superintegrable Hamiltonian systems: geometry and perturbations. Acta. Appl. Math. 87, 93-121 (2005)

Ferrer, S.: A unified treatment for some ring-shaped potentials as a generalized 4-D isotropic oscillator. Monografías Real Acad. Ciencias Zaragoza. 30, 11-21 (2006)

Grosche, C.: Coulomb potentials by path integration. Fortschr. Phys. 40, 695-737 (1992)

Hartmann, H.: Die Bewegung eines Körpers in einen ringförmigen Potentialfeld. Theor. Chim. Acta. 24, 201-206 (1972)

Hadjidemetriou, John D.: On periodic orbits and resonance in extrasolar planetary systems. Celest. Mech. Dyn. Astron. 102, 69-82 (2008)

Ikeda, M., Miyachi, Y.: On the mathematical structure of the symmetry of some simple dynamical systems. Matematica Japoniae. 15, 127-142 (1971)

Kibler, M., Lamot, G.-H., Winternitz, P.: Classical trajectories for two ring-shaped potentials. Int. J. Quantum Chem. 43, 625 (1992)

Kibler, M., Mardoyan, L.G., Pogosyan, G.S.: On a Generalized Kepler-Coulomb System: interbasis expansions. Int. J. Quantum Chem. 52, 1301 (1994)

Kibler, M., Mardoyan, L.G., Pogosyan, G.S.: On a Generalized Oscillator System: interbasis expansions. Int. J. Quantum Chem. 63, 133-148 (1996)

Kibler, M., Négadi, T.: Motion of a particle in a ring-shaped potential: an approach via a nonbijective canonical transformation. Int. J. Quantum Chem. 26, 405-410 (1984)

Kibler, M., Winternitz, P.: Dynamical invariance algebra of the Hartmann potential. J. Phys. A Math. Gen. 20, 4097-4108 (1987)

Kibler, M., Winternitz, P.: Periodicity and quasi-periodicity for super-integrable Hamiltonian Systems. Phys. Lett. A 147, 338-342 (1990)

López, C., Martínez, E., Rañada, M.F.: Dynamical symmetries, non-Cartan symmetries and superintegrability of the n-dimensional harmonic oscillator. J. Phys. A Math. Gen. 32, 1241-1249 (1999)

Makarov, A.A., Smorodinsky, J.A., Valiev, Kh., Winternitz, P.: A systematic search for nonrelativistic systems with dynamical symmetries. Nuovo Cimento A 52, 1061 (1967)

Mardoyan, L.: The generalized MIC-Kepler system, arXiv:quant-ph/0306168 v2 27 Jun (2003)

Palacián, J.: Dynamics of a satellite orbiting a planet with an inhomogeneous gravitational field. Celest. Mech. Dyn. Astron. 98, 219-249 (2007)

Poincaré, H.: Les Méthodes Nouvelles de la Mécanique Céleste, IX, 3 vols. Gauthier-Villars, Paris (1892)

Quesne, C.: A new ring-shaped potential and its dynamical invariance algebra. J. Phys. A Math. Gen. 21, 3093 (1988)

Stiefel, E.L., Scheifele, G.: Linear and regular celestial mechanics. Springer, Berlin (1971)

Synge, J.L.: Classical dynamics. Handbuch der Physik III-1. Springer, Berlin (1960) 